\title{
ON HOMEOMORPHISMS OF CERTAIN INFINITE DIMENSIONAL SPACES
}

\author{
BY \\ RAYMOND Y. T. WONG( $\left.{ }^{1}\right)$
}

1. Introduction. All spaces concerned are taken to be separable metric. In this paper we prove various properties of homeomorphisms on $l_{2}$ and certain infinite product spaces, in particular, the Hilbert cube $I^{\infty}$ and $s$ (the countable infinite product of lines).

It has been shown in [5] and [6] by V. Klee that each homeomorphism on $I^{\infty}$ (or on $l_{2}$ ) is isotopic to the identity mapping by means of into-homeomorphisms. He raised the question whether into-homeomorphisms can be replaced by selfhomeomorphisms. Results in this paper give each of his questions a positive answer. We prove that any homeomorphism on a space such as $I^{\infty}, s$, or $l_{2}$ is isotopic to the identity mapping. (Note that our definition of isotopy requires self-homeomorphisms. See 3.1.) In fact stronger theorems are obtained for homeomorphisms on spaces $I^{\infty}, s$, and $l_{2}$. Namely, any homeomorphism on each of these spaces is stable. (For definition, see $\$ 4$. In $\$ 4$ we prove stability for homeomorphism on $s$ and $l_{2}$. R. D. Anderson recently asserted the result for $I^{\infty}$ [3].) It is easy to see (by a method of Alexander) that a homeomorphism on $I^{\infty}$ (or $s$ ) is isotopic to the identity mapping if it is stable.

2. Notation. (1) If $X$ is a space, by a homeomorphism on $X$ (=self-homeomorphism) is meant a homeomorphism of $X$ onto itself.

(2) If $X$ is a space, by $X^{n}$ is meant the finite product space $\prod_{i=1}^{n} X_{i}$, where $X_{i}=X$ and by $X^{\infty}$ is meant the infinite product space $\prod_{i=1}^{\infty} X_{i}$ where $X_{i}=X$.

(3) $J, J^{\circ}, I$, and $I^{\circ}$ will denote intervals $[-1,1],(-1,1),[0,1]$, and $(0,1)$ respectively.

(4) A mapping is a continuous function.

(5) " " will mean "is homeomorphic to"; " $\sim^{i}$ " will mean "is isotopic to."

(6) By "Hilbert cube" we mean the space $J^{\infty}$ or $I^{\infty}$ with metric $\rho(x, y)$ $=\sum_{i \geqq 1}\left|x_{i}-y_{i}\right| / 2^{i}$. Hilbert space, $l_{2}$, is the space of all square summable sequences of real numbers with $d\left(\left(x_{i}\right),\left(y_{i}\right)\right)=\left(\sum_{i=1}^{\infty}\left(x_{i}-y_{i}\right)^{2}\right)^{1 / 2}$. The space $\left(J^{\circ}\right)^{\infty}$ or $\left(I^{\circ}\right)^{\infty}$ is also denoted by $s$.

(7) $e$ will always denote the identity mapping on the corresponding space.

(8) $\pi_{n}$ and $\tau_{n}$ will denote the projecting functions of $X^{\infty}$ onto $X_{n}$ and $X^{n}$

Received by the editors August 26, 1966.

(1) The work presented here was done while the author held a National Science Foundation Grant, and it forms a part of the author's doctoral dissertation under the direction of Professor R. D. Anderson. 
respectively; that is, if $x=\left(x_{1}, x_{2}, \ldots\right) \in X^{\infty}$, then $\pi_{n}(x)=x_{n}$ and $\tau_{n}(x)$ $=\left(x_{1}, x_{2}, \ldots, x_{n}\right)$.

(9) $\varnothing=$ the empty set.

(10) $\mathrm{Bd}=$ Boundary, Int = Interior.

\section{Isotopy theorems.}

3.1. Definitions. (1) $N=$ the set of all positive integers.

(2) For any $\alpha \subset N, \pi_{\alpha}$ will denote the projecting function of $X^{\infty}$ onto $\prod_{i \in \alpha} X_{i}$; that is, if $x=\left(x_{1}, x_{2}, \ldots\right) \in X^{\infty}$, then $\pi_{\alpha}(x)=\left(x_{i}\right)_{i \in \alpha}$.

(3) For any $\alpha \subset N$, if $h$ is a homeomorphism on $\prod_{i \in \alpha} X_{i}, \bar{h}$ will denote its natural extension on $X^{\infty}$. More precisely, if $x \in X^{\infty}$, then $\bar{h}(x)$ is the point in $X^{\infty}$ such that $\pi_{\alpha}(\bar{h}(x))=h\left(\pi_{\alpha}(x)\right)$ and $\pi_{i}(\bar{h}(x))=\pi_{i}(x)$ for all $i \notin \alpha$.

(4) If $h_{1}, h_{0}$ are homeomorphisms on a space $X$, then $h_{1}$ is isotopic to $h_{0}$ if there is a mapping $H$ of $X \times I$ onto $X$ such that $\left.H\right|_{X \times 1}=h_{1},\left.H\right|_{X \times 0}=h_{0}$ and for each $t \in I,\left.H\right|_{X \times t}$ is a homeomorphism on $X$. In this case we say that $\left\{h_{t}=\left.H\right|_{X \times t}\right\}_{t \in I}$ is an isotopy between $h_{1}$ and $h_{0}$.

(5) For any $\alpha \subset N$, a homeomorphism $h$ on $X^{\infty}$ is said to be fixed on the $\alpha$ coordinates if for each $x \in X^{\infty}$ and each $i \in \alpha, \pi_{i}(h(x))=\pi_{i}(x)$.

(6) If $h_{1}, h_{0}$ are homeomorphisms on $X^{\infty}$ and $\alpha \subset N$, an isotopy $\left\{h_{t}\right\}_{t \in I}$ between $h_{1}$ and $h_{0}$ is said to be fixed on the $\alpha$ coordinates if each $h_{t}$ is fixed on the $\alpha$ coordinates.

3.2. Property $\Phi$. A space $X$ satisfies property $\Phi$ if the homeomorphism $g$ on $X^{\infty}$ defined by $f\left(x_{1}, x_{2}, x_{3}, x_{4}, \ldots\right)=\left(x_{2}, x_{1}, x_{3}, x_{4}, \ldots\right)$ is isotopic to the identity mapping.

Let $\phi_{n}$ be the homeomorphism on $X_{n} \times X_{n+1}$ such that $\phi_{n}(x, y)=(y, x)$ and let $\phi_{n}$ be the natural extension of $\phi_{n}$ on $X^{\infty}$. $X$ is said to have property $\Phi^{\prime}$ if each $\phi_{n}$ is isotopic to the identity mapping under an isotopy with the property that for $n>1$, the isotopy is fixed on the first $n-1$ coordinates.

Lemma 3.1. $X$ satisfies property $\Phi$ if and only if $X$ satisfies property $\Phi^{\prime}$.

Proof. Obvious.

We shall prove several lemmas which will lead to the following theorem:

THEOREM 3.1. A necessary and sufficient condition that each homeomorphism $h$ on $X^{\infty}$ is isotopic to the identity mapping is that $X$ satisfies property $\Phi$.

Let $X$ be a space satisfying property $\Phi$ (and hence $\Phi^{\prime}$ by Lemma 3.1) and let $h$ be any homeomorphism on $X^{\infty}$. For each $n$, there is an isotopy $\left\{\phi_{n, t}\right\}_{t \in[(n-1) / n, n /(n+1)]}$ between $\phi_{n}$ and $e$ with the property that for any $n>1$ and any $t \in[(n-1) / n$, $n /(n+1)], \phi_{n, t}$ leaves the first $n-1$ coordinates fixed.

For any $a \in X$ and any $n \in N$, define mappings $a^{(n)}$ and $\tilde{\pi}_{n}$ of $X^{\infty}$ into itself as follows:

$$
\begin{aligned}
a^{(n)}\left(x_{1}, x_{2}, \ldots\right) & =\left(x_{1}, \ldots, x_{n-1}, a, x_{n}, \ldots\right) \\
\tilde{\pi}_{n}\left(x_{1}, x_{2}, \ldots\right) & =\left(x_{1}, \ldots, x_{n-1}, x_{n+1}, \ldots\right) .
\end{aligned}
$$


Lemma 3.2. If $P, P_{i} \in X^{\infty}$ such that $P_{i} \rightarrow P$, and for each $i, a_{i} \in X$, then $\tilde{\pi}_{i}\left(P_{i}\right) \rightarrow P$ and $a_{i}^{(i)}\left(P_{i}\right) \rightarrow P$.

Proof. The lemma follows since for any fixed $n$, and for any $i>n, \pi_{n}\left(\tilde{\pi}_{i}\left(P_{i}\right)\right)$ $=\pi_{n}\left(P_{i}\right)=\pi_{n}\left(a_{i}^{(i)}\left(P_{i}\right)\right)$.

For $x \in X$, denote the function $x \rightarrow\left(\pi_{n}(x)\right)^{(n)} h \tilde{\pi}_{n}(x)$ by $\tilde{h}_{n}$. The following two lemmas are evident.

LEMMA 3.3. Each $\tilde{h}_{n}$ is a homeomorphism on $X^{\infty}$ leaving the nth coordinate fixed.

LEMMA 3.4. $\tilde{h}_{n+1}=\phi_{n} \tilde{h}_{n} \phi_{n}$.

We observe that from Lemma 3.4, it follows that for any $n, \tilde{h}_{n+1}$ is isotopic to $\tilde{h}_{n}$ by means of the isotopy $\left\{h_{n, t}=\phi_{n, t} \tilde{h}_{n} \phi_{n, t}\right\}_{t \in[(n-1) / n, n /(n+1)]}$.

LEMMA 3.5. If $P, P_{i} \in X^{\infty}$ such that $P_{i} \rightarrow P$ and $\left\{f_{i}\right\}_{i \geqq 1}$ is a sequence of functions satisfying (1) each $f_{i}=\phi_{n, t}$ for some $t \in[(n-1) / n, n /(n+1)]$ and (2) for a fixed $n$, there are at most finitely many $f_{i}$ such that $f_{i}=\phi_{n, t}$. Then $f_{i}\left(P_{i}\right) \rightarrow P$.

Proof. The lemma follows since for any fixed $n$, there exists a large enough $K_{n}$ such that $\pi_{n}\left(f_{i}\left(P_{i}\right)\right)=\pi_{n}\left(P_{i}\right)$ for all $i>K_{n}$.

Lemma 3.6. If $P_{i}, P \in X^{\infty}$ such that $P_{i} \rightarrow P$, then $\tilde{h}_{i}\left(P_{i}\right) \rightarrow h(P)$.

Proof. By Lemma 3.2, $\tilde{\pi}_{i}\left(P_{i}\right) \rightarrow P$. Hence $h\left(\tilde{\pi}_{i}\left(P_{i}\right)\right) \rightarrow h(P)$. Applying Lemma 3.2 again, we get $\left(\pi_{i}\left(P_{i}\right)\right)^{(i)} h \tilde{\pi}_{i}\left(P_{i}\right) \rightarrow h(P)$. But this means $\tilde{h}_{i}\left(P_{i}\right) \rightarrow h(P)$.

LEMMA 3.7. $\tilde{h}_{1} \sim{ }^{i} h$.

Proof. Define a function $H$ of $X^{\infty} \times I$ onto $X^{\infty}$ as follows: $\left.H\right|_{X^{\infty} \times 1}=h,\left.H\right|_{X^{\infty} \times t}$ $=h_{n, t}$ where $t \in[(n-1) / n, n /(n+1)]$. (We recall that $\left\{h_{n, t}=\phi_{n, t} \tilde{h}_{n} \phi_{n, t}\right\}_{t \in[(n-1) / n, n /(n+1)]}$ is an isotopy between $\tilde{h}_{n+1}$ and $\tilde{h}_{n}$.) It suffices to show $H$ is continuous on $X^{\infty} \times 1$. Let $\left\{\left(P_{i}, t_{i}\right)\right\}_{i \geqq 1}$ be a sequence of points in $X^{\infty} \times I$ such that $\left(P_{i}, t_{i}\right) \rightarrow(P, 1)$. We may assume $t_{i}<1$ for all $i$. $H\left(P_{i}, t_{i}\right)=h_{n, t_{i}}\left(P_{i}\right)=\phi_{n, t_{i}} \tilde{h}_{n} \phi_{n, t_{i}}\left(P_{i}\right)$. Note that the sequence $\left\{\phi_{n, t_{i}}\right\}_{i}{ }_{1}$ satisfies the conditions in Lemma 3.5, hence $\phi_{n, t_{i}}\left(P_{i}\right) \rightarrow P$. By Lemma 3.6, $\tilde{h}_{n} \phi_{n, t_{i}}(P) \rightarrow h(P)$. Apply Lemma 3.5 again, $\phi_{n, t_{i}} \tilde{h}_{n} \phi_{n, t_{i}}\left(P_{i}\right) \rightarrow h(P)$ and the lemma is proved.

Proof of Theorem 3.1. The necessity is obvious. We now show the sufficiency. By Lemma 3.3, $\tilde{h}_{1}$ is the natural extension of a homeomorphism $\tilde{g}_{1}$ on $\prod_{i>1} X_{i}$. We can repeat the same argument on $\prod_{i>1} X_{i}$ and show that $\tilde{g}_{1}$ can be isotopic to a homeomorphism $\tilde{g}_{2}$ with the property that $\tilde{g}_{2}$ is the natural extension of a homeomorphism $\tilde{f}_{2}$ on $\prod_{i>2} X_{i}$. This means $\tilde{h}_{1}$ can be isotopic to $\tilde{g}_{2}$ by means of an isotopy leaving the 1 st coordinate fixed. Note that $\tilde{g}_{2}$ leaves the first two coordinates fixed. Iterating this process on $\prod_{i>2} X_{i}$, on $\prod_{i>3} X_{i}$, and so on, we see easily that $h$ is isotopic to the identity mapping.

3.3. We proceed now to show that both $J$ and $J^{\circ}$ satisfy property $\Phi$. Lemmas in the following are stated merely for $J$; similar lemmas for $J^{\circ}$ can be stated. 
Let $(\tau, \theta)$ be the polar coordinate system on the plane. Define homeomorphisms $f$ on $J^{2}, \beta, \gamma$ on the unit disk $D$ as follows:

$$
\begin{aligned}
f(r, \theta) & =(|r \cos \theta|, \theta) \text { if }-\pi / 4 \leqq \theta \leqq \pi / 4 \text { or } 3 \pi / 4 \leqq \theta \leqq 5 \pi / 4 ; \\
& =(|r \sin \theta|, \theta) \text { if } \pi / 4 \leqq \theta \leqq 3 \pi / 4 \text { or } 5 \pi / 4 \leqq \theta \leqq 7 \pi / 4 ; \\
\beta(r, \theta) & =(r, \theta+\pi) \text { and } \gamma(r, \theta)=(r, \theta+\pi / 4) .
\end{aligned}
$$

Clearly both $\beta, \gamma$ are isotopic to $e$. Denote isotopies between $\beta$ and $e$ by $\left\{\beta_{t}\right\}_{t \in I}$, between $\gamma$ and $e$ by $\left\{\gamma_{t}\right\}_{t \in I}$.

LEMMA 3.8. $F=f^{-1} \gamma f$ is a homeomorphism on $J^{2}$ such that $(1)$ if $F(x, y)=\left(x^{\prime}, y^{\prime}\right)$, then $F(y, x)=\left(-x^{\prime}, y^{\prime}\right)$ and $(2) F \sim^{i} e$.

Proof. We omit the straightforward proof of this lemma.

LEMMA 3.9. If $\omega$ is the homeomorphism on $J^{2}$ such that $\omega(x, y)=(-x,-y)$, then $\omega \sim i e$.

Proof. $\omega=f^{-1} \beta f$ and $\left\{f^{-1} \beta_{t} f\right\}_{t \in I}$ is the necessary isotopy.

LEMMA 3.10. If $\sigma$ is the homeomorphism on $J_{1}$ such that $\sigma(x)=-x$, then $\bar{\sigma} \sim^{i} e$ on $J^{\infty}$.

Proof. For each $n$, define $\omega_{n}$ on $J_{n} \times J_{n+1}$ by $\omega_{n}(x, y)=(-x,-y)$ and let $\left\{\Psi_{n, t}\right\}_{t \in[(n-1) / n, n /(n+1)]}$ be an isotopy between $\omega_{n}$ and $e$ on $J_{n} \times J_{n+1}$. Let

$$
\bar{h}_{n}=\bar{\omega}_{n} \cdots \bar{\omega}_{2} \bar{\omega}_{1} .
$$

Then $\bar{h}_{1}$ is isotopic to $e$ on $J^{\infty}$ by $\left\{h_{1, t}=\Psi_{1, t}\right\}_{t \in[0,1 / 2]}$ and for $n>1, \bar{h}_{n}$ is isotopic to $\bar{h}_{n-1}$ by $\left\{h_{n, t}=\Psi_{n, t} \bar{h}_{n-1}\right\}_{t \in[(n-1) / n, n /(n+1)]}$. Now define a mapping $H$ of $J^{\infty} \times I$ onto $J^{\infty}$ by $\left.H\right|_{J^{\infty} \times t}=h_{n, t}$ if $t \in[(n-1) / n, n /(n+1)]$ and $\left.H\right|_{J^{\infty} \times 1}=\bar{\sigma}$.

THEOREM 3.2. Any homeomorphism on the Hilbert cube is isotopic to the identity mapping.

Proof. By virtue of Theorem 3.1, it suffices to show that $J$ satisfies property $\Phi$. Let $g$ be the homeomorphism on $J^{\infty}$ defined by

$$
g\left(x_{1}, x_{2}, x_{3}, x_{4}, \ldots\right)=\left(x_{2}, x_{1}, x_{3}, x_{4}, \ldots\right),
$$

and let $F, \sigma$ be defined as before. Clearly $g=\bar{F}^{-1} \bar{\sigma} \bar{F}$. Then by Lemmas 3.8, 3.10, $g \sim i e$.

Similarly we can show that $J^{\circ}$ satisfies property $\Phi$, hence

THEOREM 3.3. Any homeomorphism on $s$ is isotopic to the identity mapping.

THEOREM 3.4. Any homeomorphism on $l_{2}$ is isotopic to the identity mapping.

Proof. This is an immediate consequence of the fact $l_{2} \sim s$ [2] and of Theorem 3.3. 
4. Stable homeomorphisms. A homeomorphism $h$ on a space $X$ is stable (in the sense of Brown-Gluck) if $h$ can be written as a composition of finitely many homeomorphisms on $X$ each of which is the identity on some open set in $X$. $s$ will denote the space $\left(I^{\circ}\right)^{\infty} . K_{1}$ will denote the set $\left\{x \in I^{\infty}: \pi_{1}(x)=1\right\}$ and $H$ will denote the space $[0,2] \times \prod_{i>1} I_{i}$, where each $I_{i}=I$. Our main result is: Any homeomorphism on $s$ or $l_{2}$ is stable. It is easy to see (as will be shown in Corollary 4.3) that (by means of Alexander's method which was originally used for $n$-cells) a homeomorphism on $s$ is isotopic to the identity mapping if it is stable. For further discussion of stable homeomorphisms on manifolds, refer to Brown-Gluck [4].

THEOREM 4.1. $s \cup K_{1} \sim s$.

THEOREM 4.2. If $K$ is a compact subset in $s$ and $h$ is a homeomorphism of $K$ into $s$, then $h$ can be extended to a stable homeomorphism $\tilde{h}$ on $s$.

For the proof of Theorem 4.1, refer to [1]. A theorem like Theorem 4.2 was proved by Klee [7] in a somewhat different context (without stressing stability). Later on Theorem 4.2 was also proved by R. D. Anderson using Klee's method [1]. Note that in Anderson's paper, stability of the homeomorphism $\tilde{h}$ was not explicitly proved, but it was explicitly observed that stability can be easily achieved for the homeomorphisms considered there.

COROLlaRY 4.1. If $s^{\prime} \sim s$, then any homeomorphism $h^{\prime}$ from a compact subset $K^{\prime}$ of $s^{\prime}$ into $s^{\prime}$ can be extended to a stable homeomorphism $\tilde{h}^{\prime}$ on $s^{\prime}$.

Proof. Let $f$ be a homeomorphism of $s^{\prime}$ onto $s$, and let $h=f h^{\prime} f^{-1} . h$ is a homeomorphism of $f\left(K^{\prime}\right)$ into $s$, hence can be extended to a stable homeomorphism $\tilde{h}$ on $s$. Write $\tilde{h}=f_{n} \cdots f_{2} f_{1}$, where each $f_{i}$ is a homeomorphism on $s$ which is the identity on some open set in $s$. Then define

$$
\tilde{h}^{\prime}=f^{-1} \tilde{h} f=f^{-1} f_{n} \cdots f_{2} f_{1} f=\left(f^{-1} f_{n} f\right) \cdots\left(f^{-1} f_{2} f\right)\left(f^{-1} f_{1} f\right) .
$$

COROLlaRY 4.2. If $h$ is a homeomorphism on $s \cup K_{1}$ and $K$ is a compact subset in $s \cup K_{1}$, then there exists a stable homeomorphism $f$ on $s \cup K_{1}$ such that fh is the identity on $K$.

Proof. $\left.h\right|_{K}$ is a homeomorphism of $K$ into $s \cup K_{1}$, hence by Theorem 4.1 and Corollary $4.1,\left.h\right|_{K}$ can be extended to a stable homeomorphism $g$ on $s \cup K_{1}$. Then let $f=g^{-1}$.

LEMMA 4.1. If $X, Y$ are spaces such that $X \sim Y$, then every homeomorphism on $X$ is stable if and only if every homeomorphism on $Y$ is stable.

Proof. Obvious, by means of the method used to prove Corollary 4.1.

LEMMA 4.2. If for each $i, i=1,2, \ldots, n, h_{i}$ is a homeomorphism on $X$ which is isotopic to the identity mapping, then $h=h_{n} \cdots h_{2} h_{1}$ is a homeomorphism on $X$ such that $h$ is isotopic to the identity mapping. 
Proof. Obvious.

THEOREM 4.3. Any homeomorphism $h$ on $s$ is stable.

Proof. By virtue of Theorem 4.1 and Lemma 4.1, it suffices to show that any homeomorphism on $s \cup K_{1}$ is stable. By Corollary 4.2, there is a stable homeomorphism $f$ on $s \cup K_{1}$ such that $f h$ is the identity on $K_{1}$. Hence there exists an open set $V$ in $s \cup K_{1}$ and a real number $r$ such that $\sup \left\{\pi_{1}(V \cup f h(V))\right\}<r<1$. Let $\varphi$ be the extension of $f h$ onto $H^{\prime}=s \cup[1,2] \times \prod_{i>1} I_{i}$ by taking $\varphi$ as the identity outside of $s \cup K_{1}$. Let $\alpha$ be a homeomorphism on [0,2] such that $\alpha$ is the identity on $[0, r]$ and $\alpha(1)=3 / 2$. Define a homeomorphism $g$ on $H$ by $g\left(x_{1}, x_{2}, \ldots\right)=\left(\alpha\left(x_{1}\right), x_{2}, \ldots\right)$. Then $\theta=\left.g^{-1} \varphi g\right|_{s \cup K_{1}}$ is a homeomorphism on $s \cup K_{1}$. Clearly $\theta$ is the identity on some neighborhood of $K_{1}$ and $\theta^{-1}(f h)$ is the identity on $V$. But $f h=\theta\left[\theta^{-1}(f h)\right]$, hence $h=f^{-1} \theta\left[\theta^{-1}(f h)\right]$, a finite composition of stable homeomorphisms. Therefore $h$ is stable.

THEOREM 4.4. Any homeomorphism on $l_{2}$ is stable.

This is an immediate consequence of the fact that $l_{2} \sim s$ [2] and of Lemma 4.1.

Corollary 4.3. Any homeomorphism $h$ on $s$ is isotopic to the identity mapping.

Proof. $h$ is stable by Theorem 4.3. Hence, by Lemma 4.2, it suffices to prove the theorem for the case that $h$ leaves some open set $V$ fixed. We now use Alexander's method applied to $s$. For some large number $n$, there is an open set $W$ in $\left(I^{\circ}\right)^{n+1}$ such that

(1) $W=\prod_{i=1}^{n}\left(a_{i}, b_{i}\right) \times\left(a_{n+1}, 1\right)$ where for each $i \leqq n, 0<a_{i}<b_{i}<1$ and $0<a_{n+1}$ $<1$ and

(2) $W \times \prod_{i>n+1} I_{i}^{\circ} \subset V$.

Let $\bar{W}$ be the closure of $W$ in $I^{n+1}, \operatorname{Int}(\bar{W})$ the interior of $\bar{W}$ in $I^{n+1}$ and let $0=(0,0, \ldots) \in I^{n+1}$. There exists a positive number $K$ such that $[0,1 / K]^{n+1} \cap \bar{W}$ $=\varnothing$. For each $x=t / K \in[0,1 / K]$, let $Q_{t}=[0, x]^{n+1}$. Let $\operatorname{Bd}(\bar{W}), \operatorname{Bd}\left(Q_{t}\right)$ denote the boundaries of $\bar{W}$ and $Q_{t}$ in $I^{n+1}$ respectively. Evidently there is a mapping $H$ of $I^{n+1} \times I$ onto $I^{n+1}$ such that:

(1) $g_{1}=\left.H\right|_{I^{n+1} \times 1}$ is the identity mapping on $I^{n+1}$.

(2) For each $0<t \leqq 1, g_{t}=\left.H\right|_{I^{n+1} \times t}$ is a homeomorphism on $I^{n+1}$ such that $g_{t}\left(I^{n+1} \mid \operatorname{Int}(\bar{W})\right)=Q_{t}$ for $0<t \leqq \frac{1}{2}$.

(3) $g_{t}(0)=0$ for all $t \in I$ and $\left.H\right|_{I^{n+1} \times 0}\left(I^{n+1} \mid \operatorname{Int}(\bar{W})\right)=0$.

Now the desired mapping $F$ from $s \times I$ onto $s$ is defined as follows: $\left.F\right|_{s \times t}$ $=\bar{g}_{t} h \bar{g}_{t}^{-1}$ for $0<t \leqq 1$ and $\left.F\right|_{s \times 0}=e$ on $s$.

\section{BIBLIOGRAPHY}

1. R. D. Anderson, Topological properties of the Hilbert cube and the infinite product of open intervals, Trans. Amer. Math. Soc. 126 (1967), 200-216.

2. - - , Hilbert space is homeomorphic to the countable infinite product of lines, Bull. Amer. Math. Soc. 72 (1966), 515-519.

3. - - - On extending homeomorphisms on the Hilbert cube, Abstract 634-56, Notices Amer. Math. Soc. 13 (1966), 375. 
4. M. Brown and H. Gluck, Stable structures on manifolds. I, Ann. of Math. (2) 79 (1964), 1-17.

5. V. Klee, Convex bodies and periodic homeomorphisms in Hilbert space, Trans. Amer. Math. Soc. 74 (1953), 36.

6. - Homogeneity of infinite-dimensional parallelotopes, Ann. of Math. (2) 66 (1957), 454-460.

7. - Some topological properties of convex sets, Trans. Amer. Math. Soc. 78 (1955), $30-45$.

8. - A note on topological properties of normed linear spaces, Proc. Amer. Math. Soc. 7 (1956), 673-674.

Louisiana State University,

Baton Rouge, LoUISIANA

UNIVERSITY OF CALIFORNIA,

Los Angeles, California 